\title{
BMJ Open Development of a prioritisation tool for the updating of clinical guideline questions: the UpPriority Tool protocol
}

\begin{abstract}
Laura Martínez García, ${ }^{1}$ Hector Pardo-Hernandez, ${ }^{1,2}$ Ena Niño de Guzman, ${ }^{1}$ Cecilia Superchi, ${ }^{1}$ Monica Ballesteros, ${ }^{1}$ Emma McFarlane, ${ }^{3}$ Katrina Penman, ${ }^{3}$ Margarita Posso, ${ }^{1}$ Marta Roqué i Figuls, ${ }^{1}$ Andrea Juliana Sanabria, ${ }^{1}$ Anna Selva, ${ }^{4}$ Robin WM Vernooij, ${ }^{1}$ Pablo Alonso-Coello ${ }^{1,2,5}$
\end{abstract}

To cite: Martínez García L, Pardo-Hernandez H, Niño de Guzman E, et al. Development of a prioritisation tool for the updating of clinical guideline questions: the UpPriority Tool protocol. BMJ Open 2017;7:e017226. doi:10.1136/ bmjopen-2017-017226

- Prepublication history for this paper is available online To view these files please visit the journal online (http://dx.doi. org/10.1136/bmjopen-2017017226).

Received 8 April 2017 Revised 7 July 2017

Accepted 7 July 2017

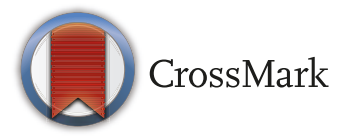

${ }^{1}$ Iberoamerican Cochrane Centre, Biomedical Research Institute Sant Pau (IIB Sant Pau), Barcelona, Spain

${ }^{2}$ CIBER of Epidemiology and

Public Health (CIBERESP),

Barcelona, Spain

${ }^{3}$ National Institute for Health and Care Excellence, Manchester, UK ${ }^{4}$ Clinical Epidemiology and Cancer Screening. Corporació Sanitaria Parc Taulí, Sabadell, Barcelona, Spain

${ }^{5}$ Clinical Epidemiology and

Biostatistics Department,

McMaster University, Hamilton, Canada

Correspondence to Dr Laura Martínez García; laura.martinez.garcia@ cochrane.es

\section{ABSTRACT}

Introduction Due to a continuous emergence of new evidence, clinical guidelines (CGs) require regular surveillance of evidence to maintain their trustworthiness.

The updating of CGs is resource intensive and time consuming; therefore, updating may include a prioritisation process to efficiently ensure recommendations remain up to date. The objective of our project is to develop a pragmatic tool to prioritise clinical questions for updating within a CG.

Methods and analysis To develop the tool, we will use the results and conclusions of a systematic review of methodological research on prioritisation processes for updating and will adopt a methodological approach we have successfully implemented in a previous experience. We will perform a multistep process including (1) generation of an initial version of the tool, (2) optimisation of the tool (feasibility test of the tool, semistructured interviews, Delphi consensus survey, external review by CG methodologists and users and pilot test of the tool) and (3) approval of the final version of the tool. At each step of the process, we will (1) calculate absolute frequencies and proportions (quantitative data), (2) use content analysis to summarise and draw conclusions (qualitative data) and (3) draft a final report, discuss results and refine the previous versions of the tool. Finally, we will calculate intraclass coefficients with $95 \%$ Cls for each item and overall as indicators of agreement among reviewers.

Ethics and dissemination We have obtained a waiver of approval from the Clinical Research Ethics Committee at the Hospital de la Santa Creu i Sant Pau (Barcelona). The results of the study will be published in peer-reviewed journal and communicated to interested stakeholders. The tool could support the standardisation of prioritisation processes for updating CGs and therefore have important implications for a more efficient use of resources in the CG field.

\section{INTRODUCTION}

Clinical guidelines (CGs) are 'statements that include recommendations intended to optimise patient care that are informed by systematic reviews (SRs) of evidence and an

\section{Strengths and limitations of this study}

To develop the tool, we will use the results and conclusions of a systematic review of methodological research on prioritisation processes for updating.

- We will adopt a methodological approach we have successfully implemented in a previous experience.

- We will collect views from clinical guidelines (CG) developers (semistructured interviews and external reviews), CG methodological experts (Delphi consensus survey) and CG users (semistructured interviews); these will allow us to pool different stakeholders' opinions about CG updating prioritisation processes

- The principal limitation of the study is that we will not perform a formal validation of the tool.

assessment of the benefits and harms of alternative care options'. ${ }^{1}$ Due to a continuous emergence of new evidence, ${ }^{23}$ CGs require regular surveillance of evidence to maintain their trustworthiness. ${ }^{45}$

Several studies have assessed length of time that CGs and their recommendations remain valid. ${ }^{4-8}$ Based on this evidence, most CG developers have adopted updating strategies based on predetermined time frames. ${ }^{9}$

An updating strategy involves different processes including the identification of new evidence; the assessment of the impact of new evidence on the current CG recommendations and whether an update is required and the update of the CG if needed. ${ }^{9}{ }^{10}$ The updating of CGs is resource intensive and time consuming. ${ }^{11}$ In the current context of restricted resources, there is a growing interest in approaches that support decision-making for updating CGs. ${ }^{12}$

We define the prioritisation process for updating of CGs as the methodology used to determine which CGs should be prioritised to ensure that resources are invested 
in updating the topics that are most relevant to different stakeholders. ${ }^{12}$ The prioritisation process includes two main stages: (1) assessment of CGs using prioritisation criteria (eg, availability of new evidence, clinical relevance or users' interest) and (2) classification of CGs in groups according to priority for updating (eg, high, medium or low relevance for updating). ${ }^{12}$

Different prioritisation processes could be implemented at different time points within an updating strategy. For example, a prioritisation process could be implemented to identify the CGs in greatest need of update (prioritisation across available CGs) ${ }^{13} 14$ or to identify the clinical questions in greatest need of update within a prioritised CG (prioritisation within a CG). ${ }^{15} 16$

Until now, there is wide variability and suboptimal reporting of the methods used to develop and implement processes to prioritise updating of CGs. ${ }^{12}$

\section{AIMS AND OBJECTIVES Primary objective}

To develop a pragmatic tool to prioritise clinical questions for updating within a CG.

\section{Secondary objectives}

- To identify the most important items required to prioritise clinical questions for updating within a CG.

- To describe each item, establish a rating scale of items and provide a guidance on how to rate them.

- To develop guidance on how to calculate and present priority scores to support decision-making for updating clinical questions within a CG.

\section{METHODS AND ANALYSIS}

To develop the UpPriority Tool, we will use the results and conclusions of a systematic review of methodological research on prioritisation processes for updating ${ }^{12}$ and will adopt a methodological approach we have successfully implemented in a previous experience. ${ }^{17} \mathrm{We}$ will perform a multistep process including (1) generation of an initial version of the tool, (2) optimisation of the tool (feasibility test of the tool, semistructured interviews, Delphi consensus survey, external review by CG methodologists and users and pilot test of the tool) and (3) approval of the final version of the tool (table 1, figure 1).

\section{Generation of the initial version of the tool Objective}

The objective is to develop the initial version of the tool (items, scoring calculation and summary report).

\section{Method}

The UpPriority Steering Group (UpSG) will participate in informal discussion and will approve the initial version of the tool.

Participants

UpSG.
OPTIMISATION OF THE TOOL

Feasibility test of the tool

Objective

The objective is to explore the feasibility and refine the initial version of the tool.

Study design

Methodological survey.

Participants

A CG developed within the Spanish National Health System Clinical Guideline Program, published within the last 2 years and with $<50$ clinical questions.

\section{Main outcome}

Time to apply the tool.

\section{Other variables}

Response rate, characteristics of participants and workplace, characteristics of clinical questions, priority scores (single item and overall items) and overall assessment of the tool (table 2).

\section{Data collection}

Two reviewers from the original Guideline Development Group (GDG) and two reviewers from the UpSG will apply the initial version of the tool. We will use online software to design the survey and collect responses (www. digestepiclin.com).

Bias

To minimise non-response bias, the survey will be available online for 1 month; weekly email reminders will be sent to reviewers. To minimise observer bias, two reviewers from outside the UpSG will apply the tool.

Study size

Convenience sample. ${ }^{18}$

Data analysis

For quantitative data, we will calculate absolute frequencies and proportions. For qualitative data, we will use content analysis to summarise and draw conclusions ( atlasti.com).$^{19}$ Questionnaires with no response in over $20 \%$ of the items will be withdrawn. We will draft a final report, discuss results and refine the initial version of the tool with the UpSG.

\section{Semistructured interviews}

Objective

The objective is to identify current practices in prioritisation processes for updating CGs and to refine the initial version of the tool.

\section{Study design}

Semistructured interviews (face-to-face, telephone or internet).

\section{Participants}

CG developers that (1) have experience in CG development and/or updating (defined as having participated in GDG and/or Guideline Updating Group (GUG) at least once in the past year) and (2) are fluent in English or Spanish. We will identify participants with the help of 


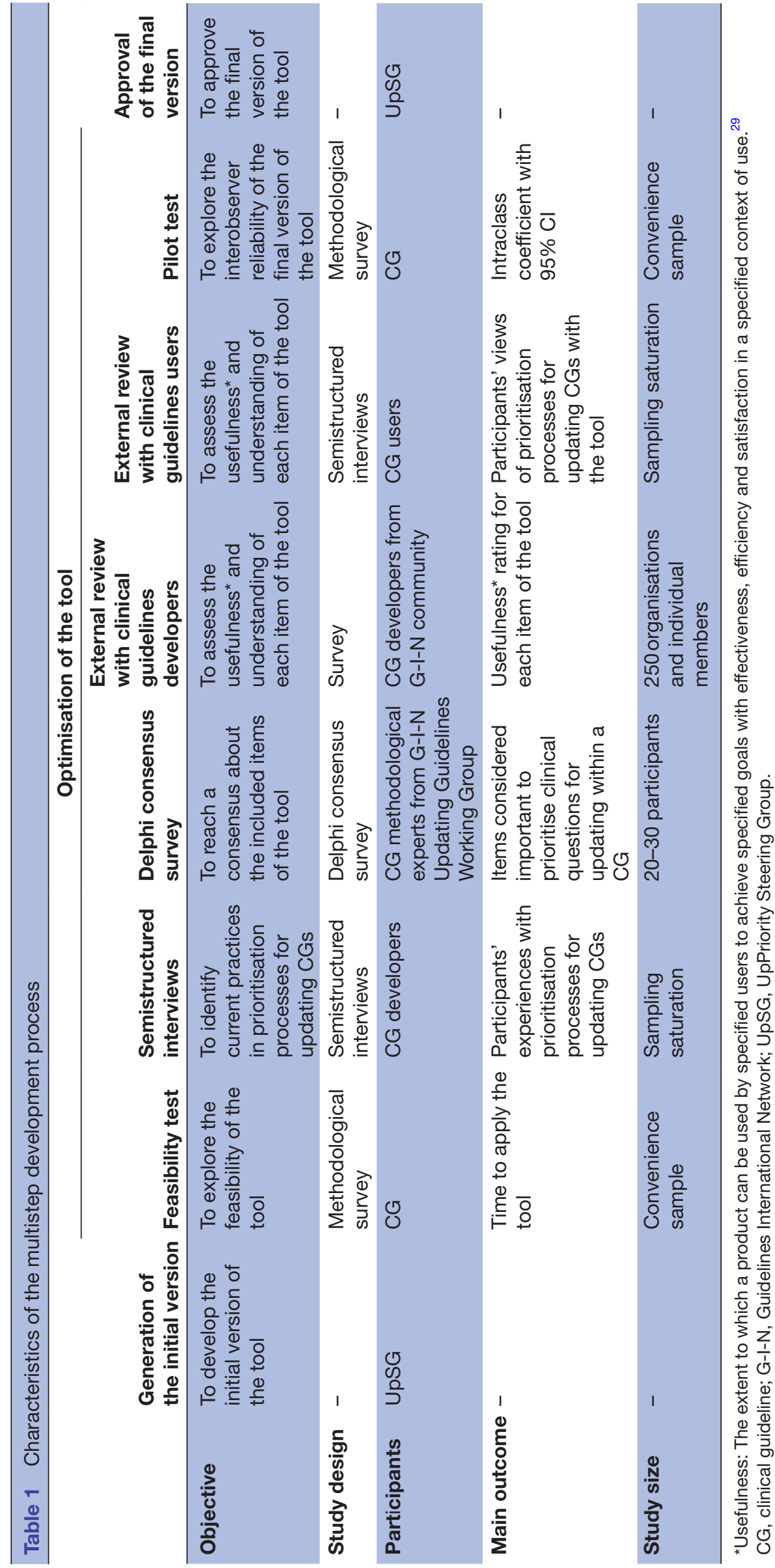




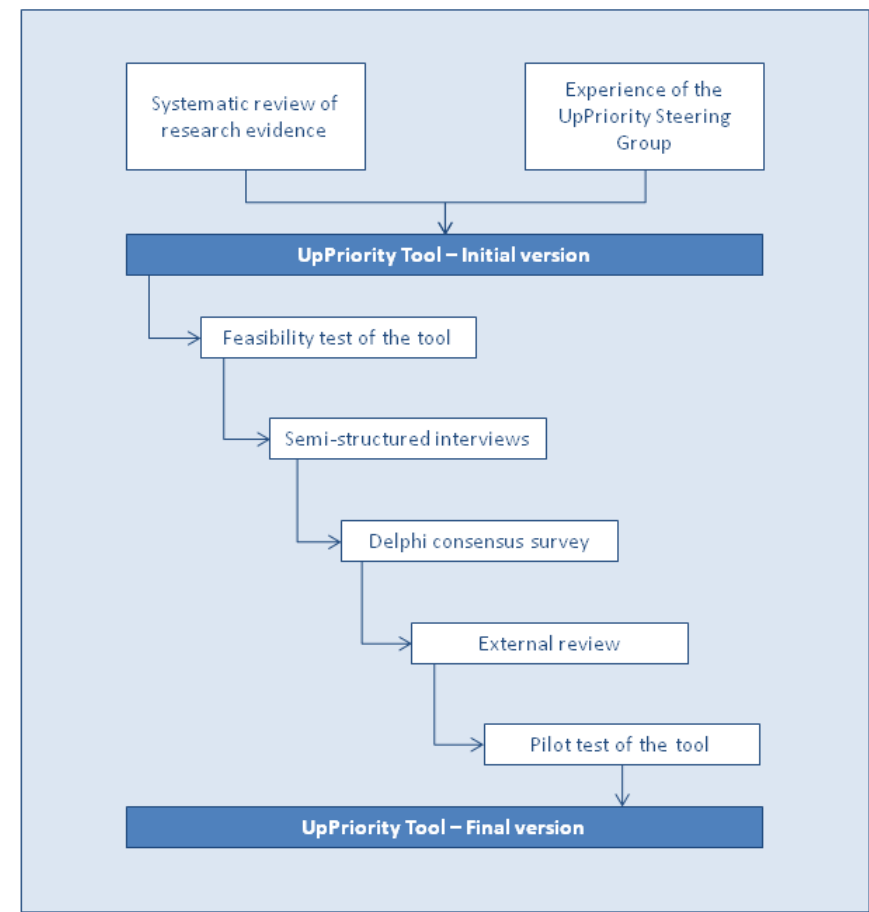

Figure 1 Multistep development process.

the UpSG. When someone does not respond or cannot participate, another contributor will be recruited.

Main outcome

Participants' experiences with prioritisation processes for updating CGs.

\section{Other variables}

Characteristics of participants and workplace, current practices in prioritisation processes for updating CGs, assessment of each item, assessment of the scoring calculation, assessment of the summary report and overall assessment of the tool (table 2).

Data collection

Interviews will be audiotaped and transcribed (each interview will last approximately 1 hour).

\section{Bias}

To minimise interviewer bias, semistructured interviews will be conducted using an interview guide.

\section{Study size}

We will recruit participants and collect data until information becomes repetitive and no new information emerges (sampling saturation)..$^{20} 21$

\section{Data analysis}

For quantitative data, we will calculate absolute frequencies and proportions. For qualitative data, we will use content analysis to summarise and draw conclusions ( atlasti.com). ${ }^{19}$ We will draft a final report, discuss results and refine the initial version of the tool with the UpSG.

\section{Delphi consensus survey}

Objective

The objective is to reach a consensus about the included items and refine the initial version of the tool.

Study design

Delphi consensus survey.

Table 2 Study variables in multistep development process

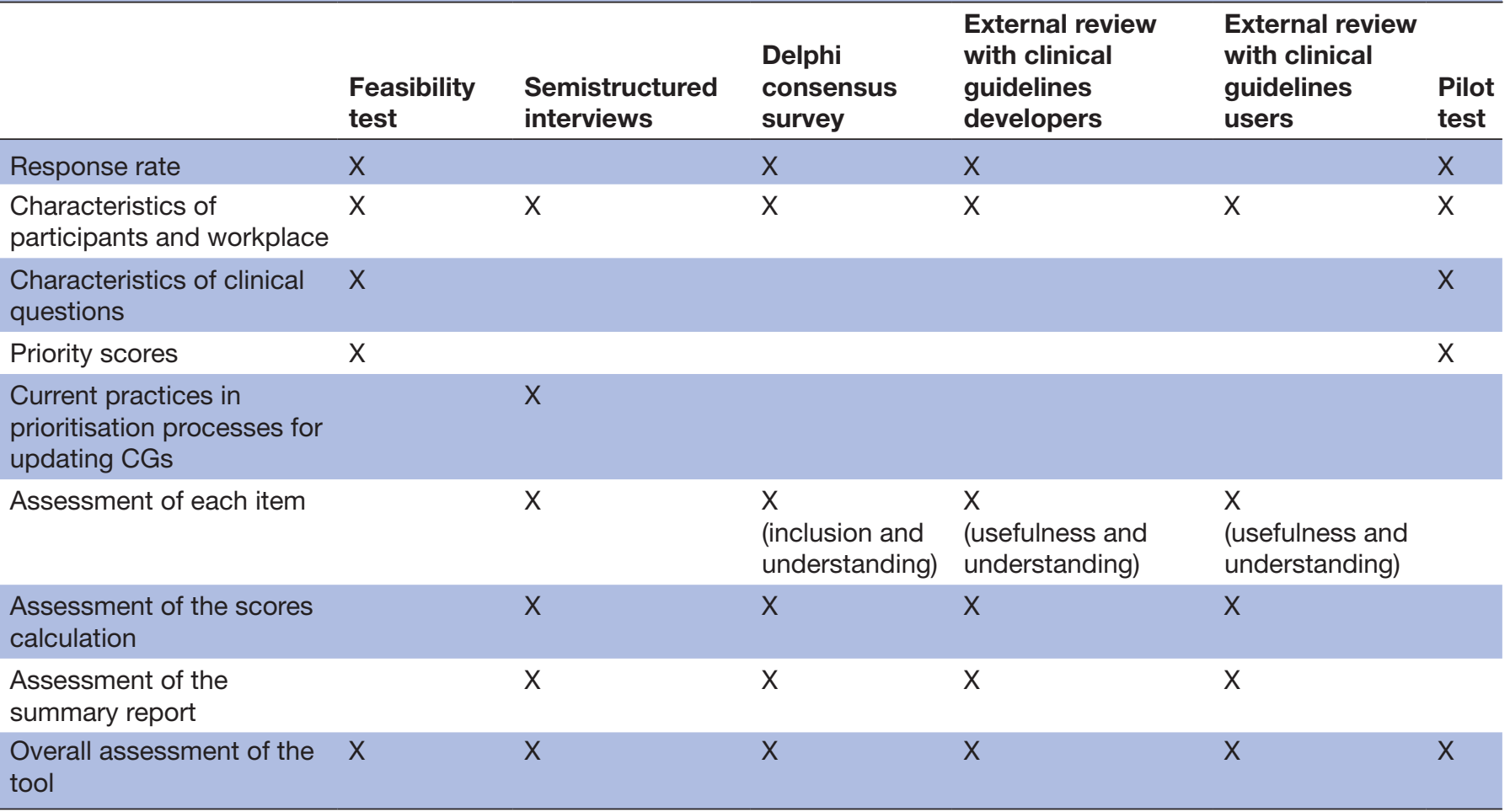

CG, clinical guideline. 
Before the first Delphi round, we will provide the results of previous methodological research to Delphi panel members.

In the first Delphi round, we will ask participants to rate whether each item should be included in the tool and its clarity using a seven-point Likert scale (1=strongly disagree and $7=$ strongly agree) ${ }^{22}$ We will calculate the median score for inclusion of each item and will classify them as (1) excluded (median score of 0-3 points), (2) review, modify and retest (median score of 4-5 points or with substantial comments) and (3) included (median score of 6 to 7 points and without substantial comments).

After each Delphi round, we will provide feedback to Delphi panel members (all responses will be anonymised prior to circulation). We will conduct additional Delphi rounds until consensus for inclusion or exclusion is reached and no more relevant comments were provided (two or three rounds, as needed).

\section{Participants}

CG methodological experts that (1) have methodological experience in CGs development and/or updating (defined as having participated in a CG technical team at least once in the past year and/or in methodological research) and (2) are fluent in English or Spanish. We will identify participants by contacting professionals associated with the Guidelines International Network (G-I-N) Updating Guidelines Working Group (http://www.g-i-n. net/working-groups/updating-guidelines) or authors of methodological research. Non-responders will not be invited to subsequent rounds.

\section{Main outcome}

Items considered important to prioritise clinical questions for updating within a CG.

\section{Other variables (per round)}

Characteristics of participants and workplace, assessment of each item (inclusion and understanding), assessment of the scoring calculation, assessment of the summary report and overall assessment of the tool (table 2).

\section{Data collection}

We will use online software to design the survey and collect responses (www.digestepiclin.com).

\section{Bias}

To minimise selection bias of Delphi panel members, all G-I-N Updating Guidelines Working Group members will be invited to participate. To minimise non-response bias, the survey will be available online for 1 month; weekly email reminders will be sent to reviewers.

\section{Study size}

Twenty to 30 participants. ${ }^{23}$

\section{Data analysis}

For quantitative data, we will calculate absolute frequencies and proportions. For qualitative data, we will use content analysis to summarise and draw conclusions ( atlasti.com).$^{19}$ Questionnaires with no response in over $20 \%$ of the items will be withdrawn. We will draft a final report, discuss results and refine the initial version of the tool with the UpSG.

\section{External review}

External review with clinical guidelines developers Objective

The objective is to assess the usefulness and understanding of each item and refine the initial version of the tool.

\section{Study design}

Survey.

\section{Participants}

CG developers that (1) have experience in CG development/updating (defined as having participated in GDG and/or GUG at least once in the past year) and (2) are fluent in English or Spanish. We will identify participants by contacting professionals associated with the G-I-N community (http://www.g-i-n.net).

\section{Main outcome}

Usefulness rating for each item of the tool.

\section{Other variables}

Characteristics of participants and workplace, assessment of each item (usefulness and understanding), assessment of the scoring calculation, assessment of the summary report and overall assessment of the tool (table 2).

\section{Data collection}

We will use online software to design the survey and collect responses (www.digestepiclin.com).

\section{Bias}

To minimise selection bias of survey participants, all G-I-N members will be invited to participate. To minimise non-response bias, the survey will be available online for 1 month; weekly email reminders will be sent to reviewers. Furthermore, the questionnaire will be pilot tested to improve wording and layout.

\section{Study size}

Currently, about 250 organisations and individual members are registered in the G-I-N community (http:/ / www.g-i-n.net/membership/members-around-theworld).

\section{Data analysis}

For quantitative data, we will calculate absolute frequencies and proportions. For qualitative data, we will use content analysis to summarise and draw conclusions ( atlasti.com).$^{19}$ Questionnaires with no response in over $20 \%$ of the items will be withdrawn. We will draft a final report, discuss results and refine the initial version of the tool with the UpSG.

\section{External review with clinical guidelines users \\ Objective}

The objective is to assess the usefulness and understanding of each item and refine the initial version of the tool. 


\section{Study design}

Semistructured interviews (face-to-face, telephone or internet).

\section{Participants}

CG users (defined as healthcare professionals that use CGs on a regular basis) who are fluent in English or Spanish. We will identify participants with the help of the UpSG. When someone does not respond or cannot participate, a new contributor will be recruited.

\section{Main outcome}

Participants' views of prioritisation processes for updating CGs with the tool.

\section{Other variables}

Characteristics of participants and workplace, assessment of each item (usefulness and understanding), assessment of the scoring calculation, assessment of the summary report and overall assessment of the tool (table 2).

\section{Data collection}

Interviews will be audiotaped and transcribed (each interview will last approximately 1 hour).

\section{Bias}

To minimise interviewer bias, semistructured interviews will be conducted using an interview guide.

\section{Study size}

We will recruit participants and collect data until information becomes repetitive and no new information emerges (sampling saturation)..$^{2021}$

\section{Data analysis}

For quantitative data, we will calculate absolute frequencies and proportions. For qualitative data, we will use content analysis to summarise and draw conclusions ( atlasti.com). ${ }^{19}$ We will draft a final report, discuss results and refine the initial version of the tool with the UpSG.

\section{Pilot test of the tool \\ Objective}

The objective is to explore the interobserver reliability of the final version of the tool and refine the initial version of the tool.

\section{Study design}

Methodological survey.

\section{Participants}

A CG developed within the Spanish National Health System Clinical Guideline Programme, published within the last 2 years and with $<50$ clinical questions.

\section{Main outcome}

Intraclass coefficient (ICG) with 95\% CI for each item and overall.

\section{Other variables}

Response rate, characteristics of participants and workplace, characteristics of clinical questions and priority scores (single item) and overall assessment of the tool (table 2).

\section{Data collection}

Two reviewers from the original GDG and two reviewers from the UpSG will apply the initial version of the tool. We will use online software to design the survey and collect responses (www.digestepiclin.com).

\section{Bias}

To minimise non-response bias, the survey will be available online for 1 month; weekly email reminders will be sent to reviewers. To minimise observer bias, two reviewers from outside the UpSG will apply the tool.

\section{Study size}

Convenience sample; the results of the pilot test will inform the sample size calculation for a subsequent main study. ${ }^{24}$

Data analysis

For quantitative data, we will calculate absolute frequencies and proportions. For qualitative data, we will use content analysis to summarise and draw conclusions ( atlasti.com). ${ }^{19}$ Questionnaires with no response in over $20 \%$ of the items will be withdrawn. We will calculate the ICC with $95 \%$ CI for each item and overall as an indicator of agreement among reviewers. According to the scale proposed by Landis and Koch, the degree of agreement between 0.00 and 0.20 is poor, from 0.21 to 0.40 is fair, from 0.41 to 0.60 is moderate, from 0.61 to 0.80 is substantial and from 0.81 to 1.00 is almost perfect. ${ }^{25}$ We will draft a final report, discuss results and refine the initial version of the tool with the UpSG.

\section{APPROVAL OF THE FINAL VERSION OF THE TOOL Objective}

The objective is to approve the final version of the tool (items, scoring calculation and summary report).

\section{Method}

The UpSG will participate in informal discussion and will approve the final version of the tool.

\section{Participants \\ UpSG.}

\section{ETHICS AND DISSEMINATION}

We have obtained a waiver of approval from the Clinical Research Ethics Committee at the Hospital de la Santa Creu i Sant Pau (Barcelona, Spain), since this study will not involve patients or biological samples.

The results of the study will be published in peer-reviewed journal and communicated to interested stakeholders (eg, via international conferences, electronic bulletin or website).

We will develop the UpPriority tool through a comprehensive development process, including the use of previous methodological evidence, ${ }^{12} 17$ feasibility testing of the tool and engagement of the international CG community (semistructured interviews, Delphi consensus survey and external review) and finally a pilot testing of the tool. 
Previous SRs on CG updating strategies found limited evidence on processes that could inform the decision of which CGs should be prioritised for updating. ${ }^{91026}$ There are, nevertheless, new studies that underscore the relevance of the prioritisation process in CG updating, ${ }^{13} 27$ coinciding with a growing interest among developers to shift from developing to updating CGs. ${ }^{28}$

We recently systematically reviewed the available evidence on strategies to prioritise the updating of SRs, health technology assessments and CGs. ${ }^{12}$ We observed that there is wide variability and suboptimal reporting of the methods used to develop and implement such prioritisation processes. Therefore, developers may have difficulties selecting and implementing a prioritisation method to optimise the updating process of CGs.

Agbassi et $a l^{13}$ implemented an annual step-by-step prioritisation process of CGs for updating. ${ }^{13}$ The authors reviewed CGs using two questionnaires; the process requires evidence search, evidence review and review approval. ${ }^{13}$ We will build our proposal on this process while addressing some of its shortcomings. Following a comprehensive development process, we will develop a pragmatic survey based tool that will likely be less resource intensive and time consuming compared with formal approaches (based on step-by-step algorithm that generally includes literature searches). We will also publish detailed and explicit guidance to allow developers to implement the tool in their institutions and to adapt it, if needed, to their specific circumstances.

We expect to develop a pragmatic tool (items, scoring calculation and summary report) that will be applicable to all clinical questions within a CG and should be easy to uptake by CG developers. The UpPriority Tool could support the standardisation of prioritisation processes for updating CGs and therefore have important implications for a more efficient use of resources in the CG field.

Contributors LMG and PAC were involved in conception and study design. LMG, $\mathrm{HPH}, \mathrm{ENG}$ and CS were involved in drafting of the first version of the article. LMG, HPH, ENG, CS, MB, EM, KP, MP, MRF, AJS, AS, RWMV and PAC were involved in critical revision of the article for important intellectual content. LMG, HPH, ENG, CS, $M B, E M, K P, M P, M R F, A J S, A S$, RWMV and PAC were involved in final approval of the article.

Funding This study has been funded by Instituto de Salud Carlos III through the project 'PI15/00325' (Cofunded by European Regional Development Fund/European Social Fund, 'Investing in your future'). Laura Martínez García is funded by a Juan Rodés contract from the Instituto de Salud Carlos III (JR15/00004). Pablo AlonsoCoello is funded by a Miguel Servet research contract from the Instituto de Salud Carlos III (MSII15/00034).

\section{Competing interests None declared.}

Ethics approval Clinical Research Ethics Committee (Hospital de la Santa Creu i Sant Pau, Barcelona, Spain).

Provenance and peer review Not commissioned; externally peer reviewed.

Open Access This is an Open Access article distributed in accordance with the Creative Commons Attribution Non Commercial (CC BY-NC 4.0) license, which permits others to distribute, remix, adapt, build upon this work non-commercially, and license their derivative works on different terms, provided the original work is properly cited and the use is non-commercial. See: http://creativecommons.org/ licenses/by-nc/4.0/ (c) Article author(s) (or their employer(s) unless otherwise stated in the text of the article) 2017. All rights reserved. No commercial use is permitted unless otherwise expressly granted.

\section{REFERENCES}

1. Steinberg E, Greenfield S, Mancher M, et al. Clinical practice guidelines we can trust. National Academies Press, 2011.

2. Bastian H, Glasziou P, Chalmers I. Seventy-five trials and eleven systematic reviews a day: how will we ever keep up? PLoS Med 2010;7:e1000326.

3. Ioannidis JP. The mass production of redundant, misleading, and conflicted systematic reviews and meta-analyses. Milbank $Q$ 2016;94:485-514.

4. Martínez García L, Sanabria AJ, García Alvarez E, et al. The validity of recommendations from clinical guidelines: a survival analysis. CMAJ 2014;186:1211-9.

5. Shekelle PG, Ortiz E, Rhodes S, et al. Validity of the Agency for Healthcare Research and Quality clinical practice guidelines: how quickly do guidelines become outdated? JAMA 2001;286:1461-7.

6. Alderson LJ, Alderson P, Tan T. Median life span of a cohort of National Institute for Health and Care Excellence clinical guidelines was about 60 months. J Clin Epidemiol 2014;67:52-5.

7. Lyratzopoulos G, Barnes S, Stegenga H, et al. Updating clinical practice recommendations: is it worthwhile and when? Int J Technol Assess Health Care 2012;28:29-35.

8. Neuman MD, Goldstein JN, Cirullo MA, et al. Durability of class I American College of Cardiology/American Heart Association clinical practice guideline recommendations. JAMA 2014;311:2092-100.

9. Vernooij RW, Sanabria AJ, Solà I, et al. Guidance for updating clinical practice guidelines: a systematic review of methodological handbooks. Implement Sci 2014;9:3.

10. Martínez García L, Arévalo-Rodríguez I, Solà I, et al. Strategies for monitoring and updating clinical practice guidelines: a systematic review. Implement Sci 2012;7:109.

11. Martínez García L, Pardo-Hernández H, Sanabria AJ, et al. Continuous surveillance of a pregnancy clinical guideline: an early experience. 2017.

12. Martínez García L, Pardo-Hernandez H, Superchi C, et al. Methodological systematic review identifies major limitations in prioritization processes for updating. J Clin Epidemiol 2017;86:11-24. Epub ahead of print.

13. Agbassi $\mathrm{C}$, Messersmith $\mathrm{H}$, McNair S, et al. Priority-based initiative for updating existing evidence-based clinical practice guidelines: the results of two iterations. J Clin Epidemiol 2014;67:1335-42.

14. Jamshidi A, Lamontagne M, Ait-kadi D, et al. Developing a comprehensive Priority-based framework for updating clinical Practice guidelines (CPGs): A systematic review and international survey. Québec, Canada: Huitième Colloque Étudiant du CIRRIS, 2016.

15. Amos Q, Chan W, Tom G. Maximising efficiency in updating guidelines through prioritisation of clinical questions [abstract]. 10th G-I-N conference. San Francisco, California, 2013.

16. Theobald S, Blanc-Vincent MP, Farsi F, et al. 1999. The identification of questions in the updating process of clinical practice guidelines for oncology [abstract]. 15th Annual Meeting of the International Society of Technology Assessment in Health Care, Edinburgh (UK)

17. Vernooij RW, Alonso-Coello P, Brouwers M, et al. Reporting items for updated clinical guidelines: checklist for the reporting of updated guidelines (CheckUp). PLoS Med 2017;14:e1002207.

18. Arain M, Campbell MJ, Cooper CL, et al. What is a pilot or feasibility study? A review of current practice and editorial policy. BMC Med Res Methodol 2010;10:67.

19. Thomas J, Harden A. Methods for the thematic synthesis of qualitative research in systematic reviews. BMC Med Res Methodol 2008;8:45.

20. Grossoehme DH. Overview of qualitative research. J Health Care Chaplain 2014;20:109-22.

21. Guetterman TC. Descriptions of sampling practices within five approaches to qualitative research in education and the health sciences. Forum Qual Soc Res 2015;16:25

22. Likert R. A technique for the measurement of attitudes. Archives of psychology 1932.

23. Akins RB, Tolson $\mathrm{H}$, Cole BR. Stability of response characteristics of a Delphi panel: application of bootstrap data expansion. BMC Med Res Methodol 2005;5:37.

24. Thabane L, Ma J, Chu R, et al. A tutorial on pilot studies: the what, why and how. BMC Med Res Methodol 2010;10:1.

25. Landis JR, Koch GG. The measurement of observer agreement for categorical data. Biometrics 1977;33:159-74. 
26. Becker M, Neugebauer EA, Eikermann M. Partial updating of clinical practice guidelines often makes more sense than full updating: a systematic review on methods and the development of an updating procedure. J Clin Epidemiol 2014:67:33-45.

27. Lord J, Willis S, Eatock $\mathrm{J}$, et al. Economic modelling of diagnostic and treatment pathways in National Institute for Health and Care Excellence clinical guidelines: the Modelling Algorithm Pathways in Guidelines (MAPGuide) project. Health Technol Assess 2013;17:1-192.
28. Baker M. Maintaining the Currency of a Large Guidelines Portfolio; Necessity is the Mother of Invention. Abstracts directory of the 13th G-I-N Conference. Philadelphia, USA, 2016. http://www.g-in.net/ document-store/g-i-n-conferences/philadelphia-2016/abstractdirectory-2016.

29. van der Weegen S, Verwey R, Tange HJ, et al. Usability testing of a monitoring and feedback tool to stimulate physical activity. Patient Prefer Adherence 2014;8:311-22. 